\title{
A Clinical Study on 299 Cases of Nasal Bone Fractures
}

\author{
Tadahiko Saiki $^{1}$ Teruhiro Ogawa ${ }^{1}$ Kazuaki Kuroda ${ }^{1}$ Satoko Miyahara ${ }^{1}$ Takuma Makino ${ }^{2}$ \\ Takahisa Koyama ${ }^{2}$ Tomoo Onoda ${ }^{3}$ \\ ${ }^{1}$ Department of Otorhinolaryngology, Himeji St Mary's Hospital, \\ Hyogo-ken, Japan \\ 2 Department of Otolaryngology-Head and Neck Surgery, Okayama \\ University Graduate School of Medicine, Dentistry and \\ Pharmaceutical Sciences, Okayama, Japan \\ ${ }^{3}$ Onoda Ear Nose Throat Clinic, Tokyo, Japan \\ Address for correspondence Tadahiko Saiki, Department of \\ Otorhinolaryngology, Himeji St Mary's Hospital, 650 Nibuno, Himeji- \\ shi, Hyogo-ken 670-0801, Japan (e-mail: saiki-t@himemaria.or.jp). \\ Int J Pract Otolaryngol 2019;2:e1-e6.
}

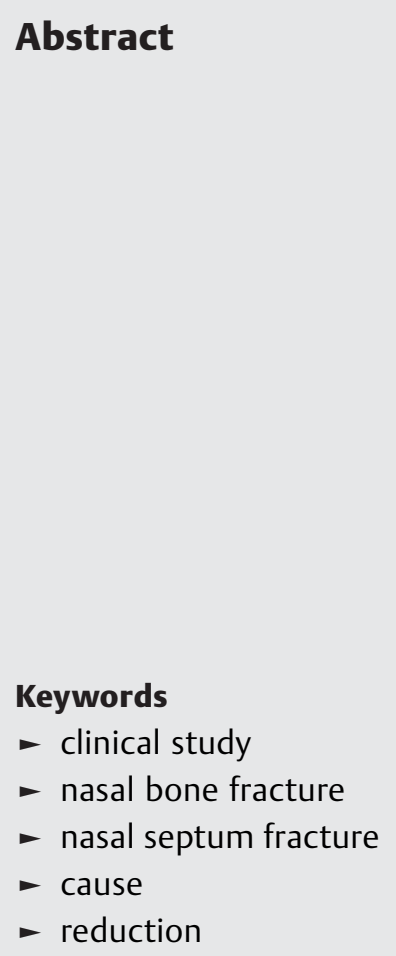

We clinically investigated 299 cases of fresh nasal bone fractures diagnosed using computed tomography (CT) scan in our hospital between January 2008 and December 2017. The ages of the patients ranged from 2 to 93 years with an average of 29.4 years. There were 207 males and 92 females. The causes of the nasal bone fractures included sports ( 93 cases), falls (80 cases), blow or injuries ( 53 cases), violence or fights (40 cases), and traffic accidents (33 cases). Males in their teenage years accounted for the majority of cases, particularly in the sports-related injuries. In these cases, the most common cause was baseball ( 32 cases), followed by soccer ( 19 cases), softball ( 15 cases), basketball ( 8 cases), and so on. In the cases of traffic accidents, nasal bone fractures were caused by bicycles ( 18 cases), cars (10 cases), motorcycles (2 cases), and so on. Elderly males and females accounted for high numbers of falls. The external appearance of the nasal bone fractures were divided into three types: the displacement type (171 cases), depressed type (96 cases), and mixed type (32 cases). CT scan revealed nasal septum fractures in 132 cases (44.2\%). Complicated facial bone fractures, apart from nasal septum fractures, were found in 35 cases (11.7\%, mainly blow-out and maxillary bone fractures). In total, 221 cases (73.9\%) underwent closed reductions of the nasal bone fracture. Of these, 206 cases (93.2\%) were treated under general anesthesia and in 131 cases (59.3\%), open reduction of the nasal septum fractures was mainly performed. In the cases of nasal bone fractures combined with nasal septum fractures, open reduction of the nasal septum fractures was helpful for the recovery of the nasal obstruction and nasal deformity.

\section{Introduction}

Nasal bone fractures are the most frequently occurring facial bone fractures; however, in recent years, opportunities to experience cases of nasal bone fractures in daily practice at the department of otolaryngology clinics are decreasing as these cases are treated more often in the department of plastic surgery. This is because nasal bone fractures often involve changes in appearance and skin damage, and considering the cosmetic aspect, consultations with the department of plastic surgery have increased. However, it is important that otolar- yngologists understand the current status as specialists treating nasal diseases concerning our daily clinical practice.

Here, we report a clinical investigation of cases of nasal bone fracture experienced in our department.

\section{Material and Methods}

The subjects included 299 cases of fresh nasal fractures diagnosed by computed tomography (CT) scans in the Department of Otorhinolaryngology at Himeji St Mary's received

August 30, 2018

accepted

November 15, 2018
DOI https://doi.org/

10.1055/s-0039-1683394. ISSN 2569-1783.
Copyright $\odot 2019$ Georg Thieme Verlag KG Stuttgart · New York
License terms

(요 (1) $\Theta \circledast$ 


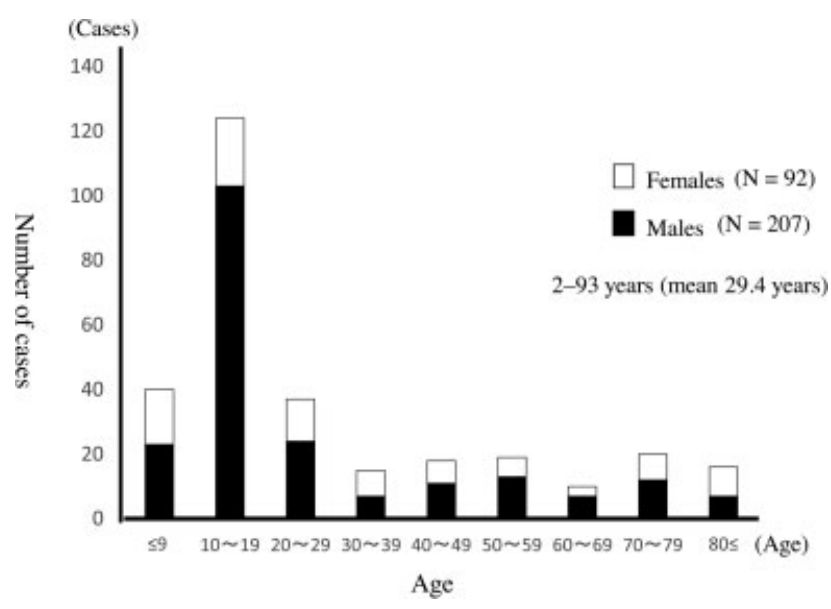

Fig. 1 Age and sex. Males in their teenage years comprised the majority of cases.

Hospital during the 10-year period between January 2008 and December 2017.

The examination items included sex and age, causes of bone fractures, fracture causes by age and sex, causes of sports-related fractures, causes of traffic accident-related fractures, frequency by classification of fracture types, directions of external force in case of assault, coexistence of other facial bone fractures, and whether or not surgical treatment was performed and the procedure types.

\section{Results}

- Age and sex

The age range of patients was 2 to 93 years, with the mean age of 29.4 years.

A total of 207 cases (69.2\%) were males and 92 cases $(30.8 \%)$ were females, and males in their teenage years accounted for the majority of cases (-Fig. 1).
- Fracture causes by age and sex

The most common causes of bone fractures were sports in 93 cases (31.1\%) followed by falls in 80 (26.8\%), blows/ trauma in 53 (17.7\%), physical violence/fights in 40 (13.4\%), and traffic accidents in 33 (11.0\%). As the causes of bone fractures by age and sex, the most common cause in men was sports (73 cases; $24.4 \%$ ), of which 62 (20.7\%) were in their teenage years. Moreover, among fall-related cases, individuals aged $\geq 70$ years comprised a large number of cases: 18 out of 45 males (40.0\%) and 15 out of 35 females (42.9\%; - Fig. 2).

- Sports-related causes

Baseball was the most common cause in 32 cases (34.4\%), followed by soccer in 19 cases (20.4\%), softball in 15 cases (16.1\%), and basketball in 8 cases (8.6\%; - Fig. 3 ). Among baseball-related cases, the cause was a hardball in 28 out of 32 cases $(87.5 \%)$, and 20 of the cases were male high school students. Among softball-related cases, the cause was a softball in 13 out of 15 cases (86.7\%), and seven of the cases were female junior high school students. Injury due to contact play was noted in 18 (94.7\%) of the soccerrelated cases, 7 (87.5\%) of the basketball-related cases, and all of the volleyball- and rugby-related cases.

- Traffic accident-related causes

Bicycle accident was the most frequent cause in 18 cases (54.5\%), followed by automobiles in 10 (30.3\%), motorcycles in 2 (6.1\%), unknown in 2 (6.1\%), and pedestrian in 1 (3.0\%). The breakdown of the cause of bicycle-related causes was 7 cases of collision with an automobile, 5 cases of falls, 5 cases falling in a gutter, and 1 case of a collision with another bicycle (-Fig. 4).

- Fracture classification by frequency Nasal bone fractures were classified into three types: displacement type by lateral external force, depressed type by anterior external force, and mixed type in which displacement type and depressed type were mixed.

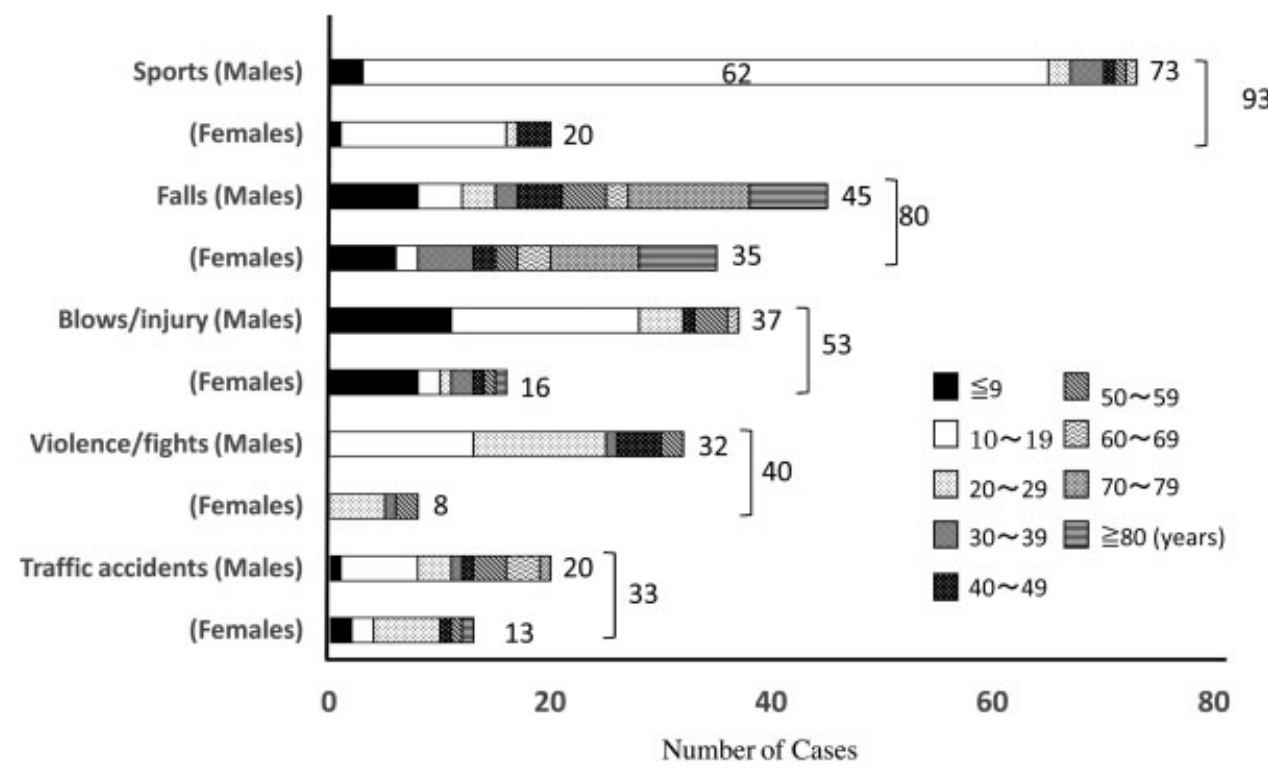

Fig. 2 Fracture causes by age and sex. Sports was the most common cause in males in their teenage years, and falls were most common in elderly persons aged $\geq 70$ years. 


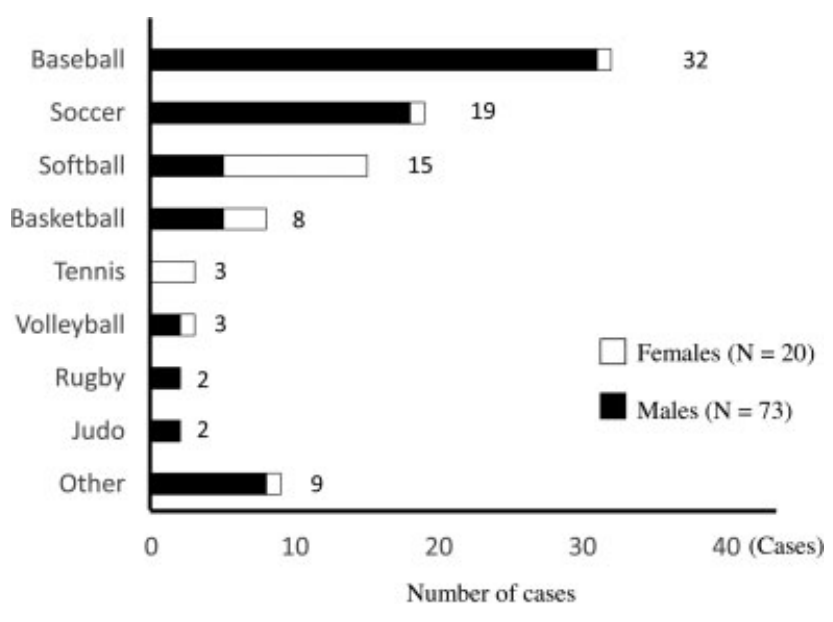

Fig. 3 Sports-related causes. The most common cause was baseball, followed by soccer and softball.

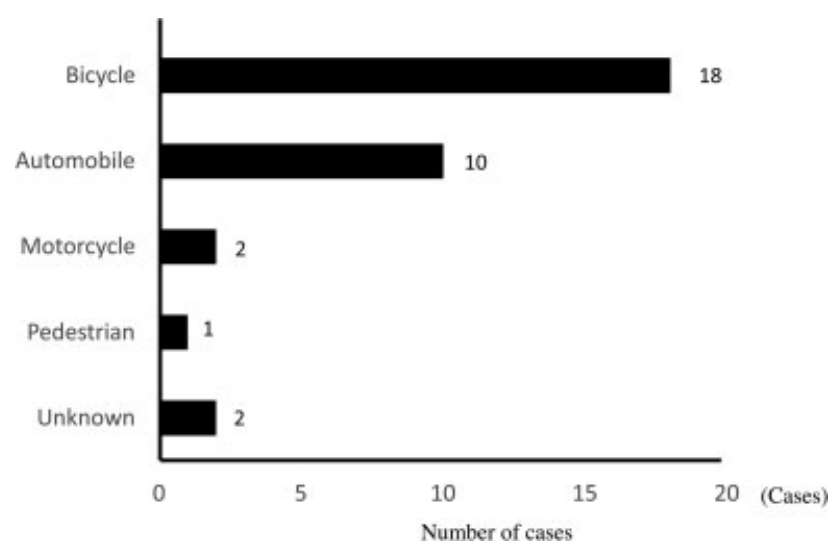

Fig. 4 Traffic accident-related causes. Bicycle was the most common cause.

Displacement type comprised 171 cases (57.2\%), depressed type 96 cases (32.1\%), and mixed type 32 cases (10.7\%; - Fig. 5). Of the cases of displacement type, external force from the right side was noted in 112 cases (55.2\%) and from the left side in 91 cases (44.8\%).

- Direction of external force in an assault

Of the 34 cases of nasal bone fractures caused by an assault, left external force was noted in 12 (35.3\%), right external force in 14 (41.2\%), and anterior external force in 8 cases (23.5\%).

- Coexistence of other facial bone fractures

CT examination revealed that 132 cases (44.2\%) had an accompanying nasal septum fracture. Of these, 49 cases (16.4\%) exhibited pneumatization in the fracture site along with damage of the nasal septum and mucous membrane, and 83 cases (27.8\%) did not show pneumatization (-Fig. 6A).

Coexisting facial bone fractures other than nasal septum fractures were found in 35 cases (11.7\%). Of these, blowout fracture only was the most common in 12 cases, followed by 11 cases of maxillary bone fractures only, and 8 cases of blow-out fracture + maxillary bone fractures ( - Fig. 6B). A total of 16 cases ( $45.7 \%$ ) were caused by fall, of these, 10 cases were elderly individuals aged $\geq 70$

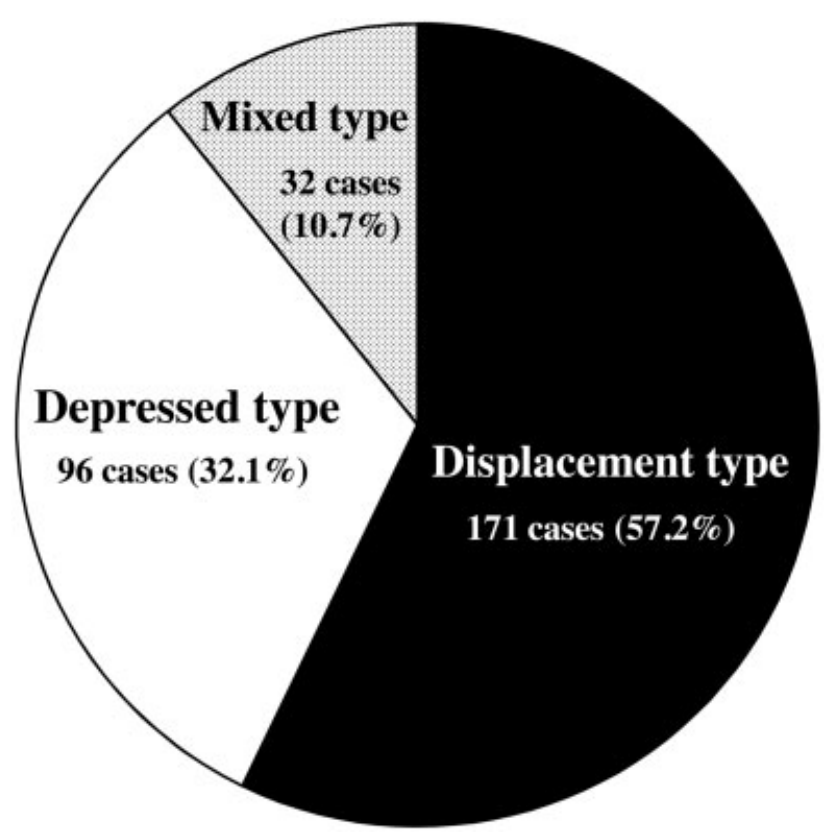

Fig. 5 Frequency by fracture classification. The most common type was displacement type, followed by depressed type and mixed type.

years. Alternatively, only three cases (8.6\%) of nasal bone fractures caused by sports, which was the most common cause, had other coexisting facial bone fractures. In addition, the fracture sites of blow-out fractures were as follows: the inner wall in 10 cases (47.6\%); the inner inferior wall in 7 cases (33.3\%); and the inferior wall in 4 cases (19.0\%).

- Whether or not surgical treatment was performed and performed procedure types

Surgical treatment was performed in 221 cases (73.9\%), of these, local anesthesia was performed in 15 cases (6.8\%) and general anesthesia in 206 cases (93.2\%). Regarding the procedures, nasal bone fracture reduction was performed in 90 cases (40.7\%) and nasal bone nasal septum fracture reduction in 131 cases (59.3\%; - Fig. 7). Reduction in nasal bone fractures was noninvasively performed in all cases, and reduction in nasal septum fractures was noninvasively performed in 12 cases and invasively (under rigid endoscopy) in 119 cases. To prevent mucosal adhesion of the olfactory cleft and to reinforce the fixation of the nasal septum after inserting a silicone sheet into the nostrils along the nasal septum, the nasal cavities were packed with tampon gauze and the dorsum was protected with a prosthesis. Intranasal tampon gauze was removed 1 week postoperatively; however, the silicone plate and nasal dorsal prosthesis were placed and worn for approximately 3 weeks postoperatively.

\section{Discussion}

The hospital is located in the northeastern part of the regional core city, and the age composition of the said medical zone shows an aging trend; however, there are no other distinctive regional characteristics, including the educational environment. In addition, our hospital also serves as an emergency 

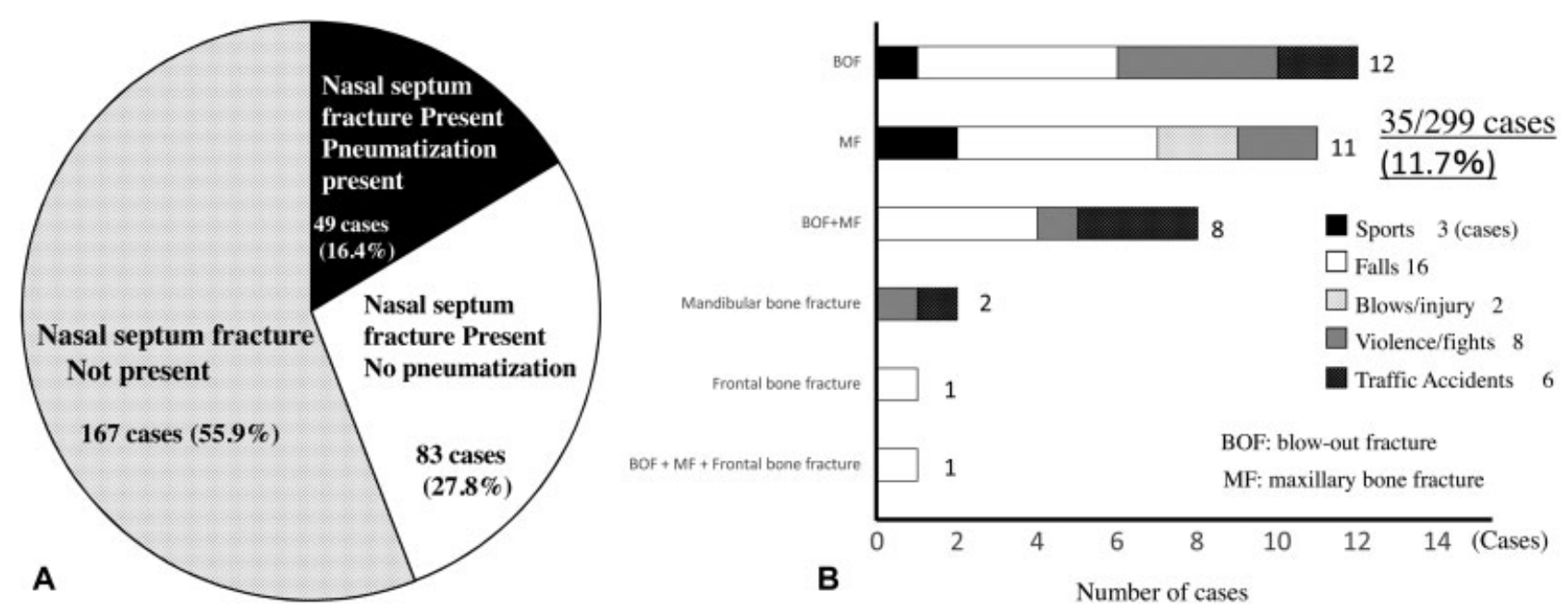

Fig. 6 (A) Nasal septum fracture cases. Based on CT scans, 44.2\% cases were complicated by nasal septal fractures. (B) Complicated by facial bone fractures (excluding nasal septum fractures). Blow-out fractures were the most common, followed by maxillary bone fractures. CT, computed tomography.

\section{Procedure}

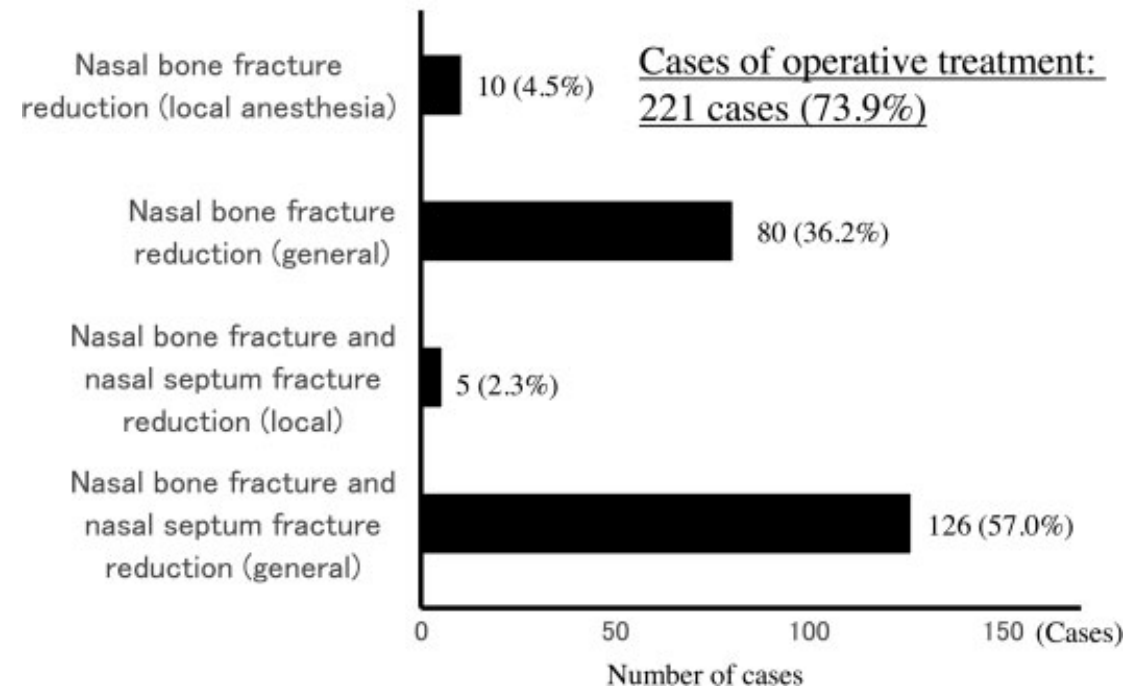

Fig. 7 Whether or not surgical treatment was performed and the procedure types. Nasal bone and nasal septal fracture reduction was more common than nasal bone fracture reduction.

medical care facility in the area, and because there is no department of plastic surgery, all nasal bone fracture cases are treated in our department. Therefore, the cases investigated in this study are considered to represent the actual state of nasal bone fractures in this medical service area.

The most common age at the time of nasal bone fracture was the teenage years and the patients aged 10 to 20 years comprised more than $70 \%$ of cases. ${ }^{1-5}$ Males are more common, with the ratio of males to females of approximately $4: 1 .^{2-4}$ Similar results were obtained in our study, probably because there are many opportunities for injury among young men. ${ }^{3}$

Most reports indicate that sports are the most common causes of bone fractures. ${ }^{5-8}$ The causes of fractures by age showed that sports are the most common cause among those in their teenage years, ${ }^{2,4}$ and in the 20 s, the number of cases of traffic accidents and violence/fights increases. It has been reported that the proportion of falls increases with age; ${ }^{4}$ moreover, the proportion of patients aged $\geq 70$ years was high in fall cases in our study. As Japan is becoming a superaging society, the number of cases of falls by elderly persons is expected to increase.

Of the sports-related causes, baseball was the most common cause, followed by other ball sports such as soccer, softball, and basketball. ${ }^{3,5-7}$ In this study, direct external force was most commonly caused by hard baseball among male high school students and softball among female junior high school students. However, injuries occurring during playing soccer, basketball, volleyball, and rugby were most commonly due to contacts with the parts such as head, elbow, and knee. Among the sports-related cases, there were many cases of injuries caused during extracurricular activities at school, and it is 
desirable that in the future, guidance for club activities is provided with prime focus on safety.

Regarding traffic accidents, the number of bicycle accidents has become the most frequent cause, reflecting the increase in popularity of bicycles in recent years. ${ }^{8}$ of our cases, bicycle-related cases were the most common, comprising more than half of all cases. In recent years, there have been cases of expensive claims for damages due to bicycles, and therefore, those who ride bicycles should comply with the traffic rules such as not riding a bicycle after consuming alcohol or riding without a light. In addition, as there were cases of falls into gutters, careful bike riding with a special caution to gutters is needed on roads with side grooves.

According to the frequencies by classification of fracture types, there are several reports that displacement types comprise approximately $70 \%$ of cases and depressed types are approximately $30 \% .{ }^{3,9,10}$ Among our cases, similar results were obtained; however, the reason for the large number of cases of displacement types is that there were many cases of external force applied laterally to the nasal bone and lateral force is more likely to cause nasal bone fracture than anterior or inferior external force. ${ }^{3,11}$ Furthermore, when there is a fracture in the upper part, the applied external force should be strong, which frequently causes a comminuted fracture. ${ }^{9}$

Although there are reports that blows with the fist tend to cause deviation to the right due to the fact that many people are right handed and use the right fist to punch, 3,12 it has also been reported that deviation to the left was more common. ${ }^{4}$ Even in our cases, left-side deviation was slightly more common than right-side deviation. The reason why deviation to the right side is not necessarily more common is that straight punch is most common although right-handedness is more common, and the injured side is determined by the timing when the person being hit attempts to divert the face.

It has been reported that coexisting nasal septum fractures were observed in 3.4 to $38.7 \%$ cases $^{2,4,5,13}$ in Japanese studies and 10 to $96.2 \%$ cases $^{14-16}$ in studies abroad. Among our cases, CT imaging confirmed coexisting nasal septum fractures in $44.2 \%$ of cases. Thus, confirming the presence or absence of complicated nasal septum fractures in nasal bone fractures is also important for treatment described later.

It is indicated that the coexisting facial bone fractures other than nasal septum fractures range from 5 to $15.7 \%$; $^{1,4,5,15,17}$ blow-out fractures were most frequent, followed by cheekbone and maxillary bone fractures. ${ }^{4,5,18}$ It appears that this is because the orbital wall and the cheekbone/maxilla are anatomically adjacent to the nasal bone. ${ }^{5}$ Among our cases, $11.7 \%$ cases involved coexisting facial bone fractures. As causes for complicated facial bone fractures, sports, which is a common cause of facial fractures among younger patients, was rare, and falls in elderly patients were highly frequent causes. This was because elderly persons tend to fall due to the decline in the sense of balance, and because their bone density is decreased, even weak external force is likely to cause other facial bone fractures.

The rate of surgical intervention for nasal bone fractures varies from 35.8 to $96.2 \%{ }^{2-4,8,10,17}$ Indications for nasal bone fracture reduction are said to include cosmetic issues due to deformation of the external nose and functional disorders such as nasal obstruction due to deformity in the nose. ${ }^{19,20}$

Regarding the method of anesthesia, reports are split on general anesthesia being mainly used ${ }^{4,7}$ and local anesthesia being mainly used. ${ }^{13}$ The advantage of general anesthesia is that there is no pain during reduction, making it possible to perform complete reduction. ${ }^{4,7}$ It has also been reported that surgery under general anesthesia is more satisfying than local anesthesia in terms of nose appearance and function in patients, and as a result, the frequency of revision surgeries can be lowered. ${ }^{21}$ However, the advantage of local anesthesia is that it can be easily and quickly performed even in an outpatient clinic and the patient can confirm the status of reduction during the procedure. ${ }^{13}$ In our department, in general, we select general anesthesia to eliminate the patient's suffering caused by pain, and to perform satisfactory reduction without hesitation.

Noninvasive reduction of nasal bone fracture is primarily performed, ${ }^{7,22}$ whereas invasive reduction of nasal septum fracture is mainly performed. When nasal septum deviation due to septum fractures accompanying the nasal bone fracture is left untreated, the deviation worsens over a long period of time, resulting in nasal obstruction and deviation of appearance also occurs; this tendency is particularly notable in pediatric patients. ${ }^{4,23}$ DeFatta et $\mathrm{al}^{24}$ reported that $60 \%$ of the cases of conservative reduction of the nasal bone and nasal septum fractures exhibited deformity of the nasal septum, whereas only $12.5 \%$ of the cases where the nasal bone was treated by a conservative reduction and open surgery was performed for the nasal septum showed deformity of the nasal septum. Based on these reports, it is recommended that nasal septum fracture reduction is performed for nasal septum deviation due to nasal septum fracture accompanying nasal bone fracture. ${ }^{4,25}$ We are also proactively performing open reductions for nasal septum fractures under rigid endoscopy, by which highly accurate reduction can be performed as it allows the surgeon to confirm the fracture site and the condition in detail with favorable mobility of the fracture site.

Kang and $\operatorname{Han}^{22}$ reported that postoperative complications occurred in $8.61 \%$ of cases; deviation was the most common, followed by saddle nose, broad nose, swelling, nasal congestion, and transient hyposmia. Another study reported that the most common complication was postoperative pain. ${ }^{18}$ Many patient satisfaction surveys have reported that 70 to $80 \%$ of the patients were satisfied; $;^{1,4,26-29}$ however, satisfaction tended to be low in severe cases with nasal septum fracture. ${ }^{28}$ Hwang et $\mathrm{al}^{15}$ reported that it should be explained to the patient that nasal deformity and deviation and obstruction of the nasal septum are postoperatively observed in $10 \%$ of the cases of nasal bone fractures. However, there are reports that the shape of the external nose after fracture reduction was improved only with follow-up observation in approximately $60 \%$ of cases at 1 month postoperatively ${ }^{29}$ and in approximately 50\% at 3 months postoperatively. ${ }^{30}$ Although there were no cases in our study that required revision surgery owing to nasal obstruction or deformity of the external nose, follow-up observation may be necessary for at least 3 months postoperatively for the symptoms to become fixed and to determine the necessity of postoperative revision surgery. 


\section{Conclusion}

- We clinically investigated 299 cases of fresh nasal bone fractures diagnosed by CT scan at the Department of Otorhinolaryngology of Himeji St Mary's Hospital over a 10-year period between January 2008 and December 2017.

- The ages of the patients ranged from 2 to 93 years (mean age: 29.4 years); 207 cases were males and 92 were females.

- The most common causes of nasal bone fractures included sports, followed by fall, blow or traumas, violence or fights, and traffic accident.

- Among the sports-related cases, baseball was the most common cause among males in their teenage years, falls among males and females aged $\geq 70$ years, and bicycle among traffic accident-related cases.

- The frequencies by classification of nasal bone fracture types were as follows: displacement type, 171 cases (57.2\%); depressed type, 96 cases (32.1\%); and mixed type, 32 cases (10.7\%).

- CT examination revealed that accompanying nasal septum fractures were noted in 132 cases (44.2\%) and other facial bone fractures in 35 (11.7\%), and blow-out fractures were the most frequent with the exception of nasal septum fractures.

- Surgical treatment was performed in 221 patients (73.9\%), of these, nasal septum reduction was also performed in 131 cases (59.3\%). Procedures were performed under general anesthesia in $93.2 \%$ cases and local anesthesia in $6.8 \%$ cases.

- Our study indicated that noninvasive reduction of nasal septum fractures should be proactively performed in the cases of nasal bone fractures complicated with nasal septum fractures.

Conflict of Interest

None declared.

\section{References}

1 Yoshida S, Okitsu T, Shoji F, et al. Clinical statistics and diagnostic imaging of nasal bone fractures. J Sendai City Hosp 1991;11:69-73

2 Nario K, Tsuruta Y, Tanaka O, et al. Clinical statistics of nasal bone fractures. Pract Otorhinolaryngol (Basel) 1992;85:565-574

3 Bandoh N, Hishii H, Kanaya T. Clinical study of nasal fractures. Pract Otorhinolaryngol (Basel) 2000;93:361-366

4 Ogawa T, Suzuki N, Okitsu T. Clinical statistics and image diagnosis of nasal bone fractures. Pract Otorhinolaryngol (Basel) 2002;95:51-61

5 Takekawa C, Sakura A, Takasu H, et al. Statistical examination of 235 nasal bone fractures-correlation between injury and severity. J Japan Soc Plast Reconstr Surg 2011;31:73-78

6 Kase M, Miwa T, Miya W, et al. A review of cases with nasomaxillofacial fractures. Otorhinolaryngology 1986;29:65-180

7 Fujitani S, Aiba M, Sugiuchi T, et al. A review of nasal bone fracture cases. Otorhinolaryngology 1987;30:39-44
8 Kon A, Suzuki H, Miyata M, et al. Statistical investigation of facial bone fractures. Niigata Igakkai Zasshi 2012;126:40-46

9 Hirota Y, Shimizu Y, Iinuma T. Image diagnosis of nasal bone fracture [in Japanese]. Nippon Jibiinkoka Gakkai Kaiho 1988;91 (04):539-546

10 Illum P, Kristensen S, Jørgensen K, Brahe Pedersen C. Role of fixation in the treatment of nasal fractures. Clin Otolaryngol Allied Sci 1983;8(03):191-195

11 Colton JJ, Beekhuis GJ. Management of nasal fractures. Otolaryngol Clin North Am 1986;19(01):73-85

12 Ichimura K, Yoshinami H. Topics otolaryngology/head and neck region sports trauma -2 nasal/paranasal. Otolaryngol Head Neck Surg 1995;67:29-38

13 Hasegawa T, Kumoi K. Clinical study of facial bone fractures. Pract Otorhinolaryngol (Basel) 2006;99:961-965

14 Rhee SC, Kim YK, Cha JH, Kang SR, Park HS. Septal fracture in simple nasal bone fracture. Plast Reconstr Surg 2004;113(01):45-52

15 Hwang K, You SH, Kim SG, Lee SI. Analysis of nasal bone fractures; a six-year study of 503 patients. J Craniofac Surg 2006;17(02): 261-264

16 Hwang K, Yeom SH, Hwang SH. Complications of nasal bone fractures. J Craniofac Surg 2017;28(03):803-805

17 Oikawa K, lizuka K, Higuchi E, et al. Clinical investigation of nasal bone fractures in our department. Otolaryngol Head Neck Surg 1998;70:94-98

18 Park HK, Lee JY, Song JM, Kim TS, Shin SH. The retrospective study of closed reduction of nasal bone fracture. Maxillofac Plast Reconstr Surg 2014;36(06):266-272

19 Fukami M. Nasal bone fractures. Otolaryngol Head Neck Surg 1997;69:60-64

20 Miyajima Y, Fukunaga H, Nakashima T. Clinical statistics on nasal injuries. Pract Otorhinolaryngol (Basel) 2003;96:955-958

21 Al-Moraissi EA, Ellis E III. Local versus general anesthesia for the management of nasal bone fractures: a systematic review and meta-analysis. J Oral Maxillofac Surg 2015;73(04):606-615

22 Kang CM, Han DG. Objective outcomes of closed reduction according to the type of nasal bone fracture. Arch Craniofac Surg 2017;18(01):30-36

23 Fry $\mathrm{H}$. The importance of the septal cartilage in nasal trauma. $\mathrm{Br} \mathrm{J}$ Plast Surg 1967;20(04):392-402

24 DeFatta RJ, Ducic Y, Adelson RT, Sabatini PR. Comparison of closed reduction alone versus primary open repair of acute nasoseptal fractures. J Otolaryngol Head Neck Surg 2008;37 (04):502-506

25 Kane NP, Kane LA. Open reduction of nasal fractures. J Otolaryngol 1978;7(02):183-186

26 Nakamaru Y, Honma A, Iizuka K. Clinical investigation of nasal bone fractures in our department. Otolaryngol Head Neck Surg 1993;65:745-748

27 Hung T, Chang W, Vlantis AC, Tong MC, van Hasselt CA. Patient satisfaction after closed reduction of nasal fractures. Arch Facial Plast Surg 2007;9(01):40-43

28 Kang CM, Han DG. Correlation between operation result and patient satisfaction of nasal bone fracture. Arch Craniofac Surg 2017;18(01):25-29

29 Lim H, Kang KR, Koh SH, Jung SW, Jung S. Postoperative changes after closed reduction of nasal fracture. J Craniofac Surg 2017;28 (07):1649-1653

30 Lee BM, Han DG. Acute bone remodeling after reduction of nasal bone fracture on computed tomography imaging. Arch Craniofac Surg 2014;15(02):63-69 